\title{
Serotonin Syndrome in a Patient on Trazodone and Duloxetine Who Received Fentanyl following a Percutaneous Liver Biopsy
}

\author{
Ryan R. Gaffney Ian R. Schreibman \\ Division of Gastroenterology and Hepatology, Penn State College of Medicine and \\ Milton S. Hershey Medical Center, Hershey, Pa., USA
}

\section{Key Words}

Serotonin syndrome $\cdot$ Percutaneous liver biopsy $\cdot$ Trazodone $\cdot$ Duloxetine $\cdot$ Fentanyl

\begin{abstract}
Serotonin syndrome is a rare but potentially life-threatening adverse drug reaction resulting from the use or overuse of serotonergic medications alone or in combination. Mild symptoms, overlapping features with similar conditions and clinician lack of awareness are the major reasons for an often missed diagnosis. Not surprisingly, this condition is significantly underreported as a potential complication of endoscopy if serotonergic medications are used periprocedurally for sedation and analgesia. Here we report the case of a patient with relapsed chronic hepatitis $C$ on antidepressant medications who developed signs and symptoms of serotonin syndrome after a percutaneous liver biopsy. Review of the patient's medication list suggested a possible interaction between her home antidepressants and the postprocedure use of fentanyl for abdominal pain. The patient required monitoring in the medical intensive care unit and stabilized after the administration of benzodiazepines and temporary discontinuation of her home medications. We propose that clinicians need to be aware of the increased risk of serotonin syndrome in the outpatient endoscopy setting, particularly with the wider use of serotonergic antidepressants now available and the repeated number of liver biopsies being performed for management of patients with chronic liver disease.
\end{abstract}


Gaffney and Schreibman: Serotonin Syndrome in a Patient on Trazodone and

Duloxetine Who Received Fentanyl following a Percutaneous Liver Biopsy

\section{Introduction}

Percutaneous liver biopsy is the most specific test currently available to assess the nature and severity of liver disease, and ultrasound guidance has allowed this technique to be performed routinely and safely in the outpatient setting [1]. Because of its invasive nature, pain is considered the most common complication, with an incidence of $35 \%$ reported in the literature [2]. Although the intensity of pain has not been specifically studied, a majority of patients have a short duration of symptoms that is considered minor by most hepatologists. The use of short-acting opioids like fentanyl for periprocedural analgesia after liver biopsy is common practice, and most patients do not require hospitalization.

Serotonin syndrome is a rare but potentially life-threatening adverse drug reaction resulting from the therapeutic use or intentional overdosage of serotonergic medications alone or in combination [3]. This condition is often described as a clinical triad of mental status changes, neuromuscular abnormalities and autonomic hyperactivity [4]. The majority of cases occur within $6 \mathrm{~h}$ of a change or initiation of a serotonergic drug. No laboratory tests can confirm the diagnosis. Instead, the presence of tremor, akathisia or clonus in the proper clinical situation should lead clinicians to consider serotonin syndrome [3]. The general principles of management include discontinuation of all serotonergic agents, supportive care with intravenous hydration and control of agitation with anxiolytics.

The list of drugs and drug combinations associated with serotonin syndrome is extensive and includes antidepressants, antiemetics, antitussive agents, antibiotics, antimigraine medications, drugs of abuse, dietary supplements and analgesics [3]. Opioids may significantly affect serotonin kinetics in the presence of other serotonergic agents [5]. Use of opioids for post-procedure pain control may therefore have significant clinical consequences, especially with the abundance of polypharmacy in current practice. The risk for misdiagnosis of this syndrome is further potentiated by the limited knowledge of multiple interactions among prescribed medications.

To our knowledge, this is the first reported case of serotonin syndrome experienced by a patient undergoing a liver biopsy. The diagnosis was made after a diligent history and physical examination and after exclusion of other diagnoses. Our patient was managed conservatively with administration of benzodiazepines and close monitoring, with a good outcome.

\section{Case Presentation}

A 59-year-old white female with chronic hepatitis C presented to our endoscopy suite for a percutaneous liver biopsy. The patient had been diagnosed with hepatitis $C$ virus genotype 1a 5 years prior and had initially been treated with a 48-week course of pegylated interferon alfa-2a (Pegasys; Genentech USA, Inc.) and ribavirin. Unfortunately she had relapsed within 6 months of stopping antiviral therapy. A percutaneous liver biopsy at that time had demonstrated active chronic hepatitis. The patient had failed a second course of antiviral therapy with pegylated interferon and ribavirin. She had subsequently developed diffuse arthralgias attributed to hepatitis $\mathrm{C}$ virus-related cryoglobulinemia. After discussing the risks and benefits of protease inhibitor-based antiviral therapy, the decision was made to perform a surveillance percutaneous liver biopsy for restaging of her disease.

The patient's home medications included valsartan $160 \mathrm{mg}$ daily for hypertension, lansoprazole $30 \mathrm{mg}$ every morning for dyspepsia, simvastatin $10 \mathrm{mg}$ daily for hyperlipidemia, and lorazepam as needed for anxiety. She was also taking trazodone $100 \mathrm{mg}$ at bedtime for insomnia and duloxetine $60 \mathrm{mg}$ daily for depression. She reported stable control of her 
Gaffney and Schreibman: Serotonin Syndrome in a Patient on Trazodone and Duloxetine Who Received Fentanyl following a Percutaneous Liver Biopsy

symptoms with these medications for several years without any adverse drug interactions. She had medication allergies to sulfa-containing compounds, acetaminophen and erythromycin. Before the procedure the patient was without any noted complaint. She was afebrile with normal vital signs (blood pressure of 120/78 mm Hg and pulse of 71 beats per minute). A percutaneous liver biopsy was performed without immediate complication using sterile technique and ultrasound guidance. The patient's vital signs remained stable during the procedure, and she was sent to the recovery suite for continued observation.

Approximately $10 \mathrm{~min}$ post-procedure, the patient developed intense right-sided abdominal and chest pain. On re-evaluation, her cardiopulmonary and abdominal examinations were benign. Chest radiography showed no evidence of pneumothorax or free air, and laboratory testing was unremarkable for acute anemia, renal insufficiency, acidemia or transaminitis. She was medicated with $50 \mu \mathrm{g}$ of intravenous fentanyl, which did not alleviate her pain. She was then administered an additional $50 \mu \mathrm{g}$ of fentanyl divided in two separate doses. Approximately $5 \mathrm{~min}$ after receiving the fentanyl the patient became agitated, diaphoretic and hypertensive. She displayed pressured speech and was in a moderate amount of distress. She was breathing spontaneously at 25 breaths per minute with a blood pressure of 198/106 mm Hg, a pulse of 82 beats per minute and an oxygen saturation of $98 \%$ on room air. Serial physical examination revealed profound flushing, diaphoresis, a soft abdomen with increased bowel sounds and mild rigidity in all four extremities. $2 \mathrm{mg}$ of intravenous lorazepam was administered with marked improvement in her vital signs and symptoms. She was admitted to the medical intensive care unit for close observation where she was made nil per os, and computed tomography imaging of her chest, abdomen and pelvis demonstrated no complications from the biopsy. She received intravenous fluids, anxiolytics and pain control with hydromorphone with good effect.

The patient remained afebrile during her brief hospital stay, with gradual resolution of her agitated state and widespread pain within $24 \mathrm{~h}$. Her blood pressures demonstrated improvement, but did not return to baseline. She was resumed on valsartan and tolerated advancement of her diet. Although no specific etiology for her abdominal and chest pain was found, review of the patient's medications suggested a possible interaction between the recently administered fentanyl and her antidepressants duloxetine and trazodone. Considering the presentation, a diagnosis of serotonin syndrome was made, and fentanyl was added to her medication allergy list. Since her depression and insomnia had been stable on duloxetine and trazodone without any previous adverse interactions, she was discharged from the hospital with instructions to restart these antidepressants. The patient was seen in follow-up 1 week after her hospital discharge and her physical exam revealed normal vital signs and no neurologic findings. Her liver biopsy results eventually showed mild inflammatory activity with fibrosis.

\section{Discussion}

Serotonin syndrome is a rare but potentially life-threatening adverse drug reaction resulting from the therapeutic use or intentional overdosage of serotonergic medications alone or in combination [3]. First described in the 1950s as an idiopathic drug reaction between iproniazid and pethidine, serotonin syndrome is a recognized clinical condition with established diagnostic criteria and predictable consequences [6]. The current literature cites an incidence of 14-16\% in patients who overdose solely on selective serotonin reuptake inhibitors, although this number likely underestimates the true number of cases reported annually 
Gaffney and Schreibman: Serotonin Syndrome in a Patient on Trazodone and

Duloxetine Who Received Fentanyl following a Percutaneous Liver Biopsy

because symptoms are often unrecognized by clinicians or perceived secondary to other disease processes $[7,8]$.

Serotonin is a monoamine neurotransmitter located centrally (central nervous system) in the brainstem raphe nuclei where it is responsible for thermoregulation and wakefulness and peripherally (peripheral nervous system) within platelets and intestinal enterochromaffin cells where it modulates smooth muscle function [3]. The pathophysiologic mechanisms for this condition center around postsynaptic hyperstimulation of 5-hydroxytryptamine $1 \mathrm{~A}$ and $2 \mathrm{~A}$ serotonin receptors in the central and peripheral nervous system, typically via the simultaneous administration of two or more drugs that enhance serotonin [9]. The list of drugs and drug combinations associated with serotonin syndrome is extensive and includes antidepressants, antiemetics, antitussive agents, antibiotics, antimigraine medications, drugs of abuse, dietary supplements and analgesics [3].

The diagnosis of serotonin syndrome is historically based on a triad of neuroexcitatory features, including altered mental status, neuromuscular abnormalities and autonomic hyperactivity [4]. The condition encompasses a wide spectrum of clinical findings that directly correlate with intrasynaptic serotonin levels, but interestingly not serum serotonin concentrations [10]. Mild cases may present only with tachycardia, diaphoresis and subtle confusion. Moderate cases, such as the patient reported here, often display frank vital sign abnormalities like hypertension, tachypnea and agitation. Many of these patients often have physical neurologic findings such as clonus or hyperreflexia. Severe cases of serotonin syndrome manifest with critical hypertension and muscle rigidity, and these patients can deteriorate rapidly into disseminated intravascular coagulation, rhabdomyolysis and shock, and become comatose [3]. Regardless of the severity, the majority of cases occur within $6 \mathrm{~h}$ of the change or initiation of a serotonergic drug, thus emphasizing the need for a high index of suspicion and immediate recognition. The general principles of management include discontinuation of all serotonergic agents, supportive care with intravenous hydration and control of agitation with anxiolytics. In some instances, the serotonin antagonist cyproheptadine may be administered.

Opioids are a mainstay of pain management, and in clinical practice they are often administered for sedation during outpatient surgeries or for immediate postoperative analgesia. Fentanyl is one of the most commonly used opioids in this ambulatory setting because of its short half-life and lack of histamine-releasing effect [11]. In recent years, the synthetic phenylpiperidine series of opioids, including fentanyl and meperidine, has been associated with serotonin syndrome through weak serotonin reuptake inhibition and direct serotonergic agonism [5]. The first reported case of serotonin syndrome involving a medication interaction with fentanyl occurred in a 65-year-old female chronically treated with citalopram for depression [12]. Within $24 \mathrm{~h}$ of initiation of a topical fentanyl patch to manage symptoms of worsening back and abdominal pain, the patient developed confusion, agitation, myoclonic jerks and hyperreflexia. In recent years, several additional case scenarios have raised awareness for the number of potential iatrogenic adverse interactions of serotonergic drugs with commonly prescribed opioids like fentanyl [13].

To date, there has been only one case of serotonin syndrome described as a complication of fentanyl use specifically in the endoscopic setting [14]. A 39-year-old woman with alcoholic cirrhosis underwent esophagogastroduodenoscopy for evaluation of hematemesis and was found to have Mallory-Weiss syndrome and grade I nonbleeding esophageal varices. She was chronically taking sertraline $100 \mathrm{mg}$ daily for depression. Pre-procedure, the patient was medicated with $50 \mu \mathrm{g}$ of fentanyl divided in two doses of $25 \mu \mathrm{g}$ each. After the esophagogastroduodenoscopy, she became somnolent and hyperthermic and developed rigidity in all four extremities, ultimately requiring intubation for airway protection. The 
Gaffney and Schreibman: Serotonin Syndrome in a Patient on Trazodone and

Duloxetine Who Received Fentanyl following a Percutaneous Liver Biopsy

diagnosis of serotonin syndrome was made because of a suspected interaction between fentanyl and sertraline.

Serotonin syndrome is a potentially deadly result of polypharmacy that has been well described in the literature for years. Mild symptoms, overlapping features between syndromes such as neuroleptic malignant syndrome and malignant hyperthermia, and clinician lack of awareness are reasons for an often missed diagnosis [13]. Our patient displayed moderate neuroexcitatory symptoms immediately after the administration of fentanyl for periprocedural pain, yet was admitted to the hospital for several hours before an accurate recognition of her condition was made. We propose that clinicians need to be aware of the increased risk of serotonin syndrome in the perioperative and outpatient endoscopic setting, especially with the wider use of selective serotonin reuptake inhibitors and the growing number of serotonergic medications available today in clinical practice. Specific patient characteristics such as anxiety level, chronic use of addictive medications and request for sedation have been identified as risk factors for analgesic use following liver biopsy. Special consideration of these factors may improve decision making and resource utilization in the care of patients with pain after liver biopsy [15]. Furthermore, gastroenterologists and hepatologists need to be aware of the increased risk of serotonin syndrome in the perioperative setting where high-dose opioids are often administered. Stringent attention must be paid to a patient's list of home medications and documented allergies. If a patient is taking multiple serotonergic medications, it is wise to avoid or use with caution the phenylpiperidine opioids, including fentanyl, for post-procedure analgesia because of their weak serotonin reuptake inhibition.

\section{References}

$\$ 1$ Copel L, Sosna J, Kruskal JB, Kane RA: Ultrasound-guided percutaneous liver biopsy: indications, risks, and technique. Surg Technol Int 2003;11:154-160.

2 Castéra L, Nègre I, Samii K, Buffet C: Pain experienced during percutaneous liver biopsy. Hepatology 1999;30:1529-1530.

3 Boyer E, Shannon M: The serotonin syndrome. N Engl J Med 2005;352:1112-1120.

4 Dunkley EJ, Isbister GK, Sibbritt D, Dawson AH, Whyte IM: The Hunter Serotonin Toxicity Criteria: simple and accurate diagnostic decision rules for serotonin toxicity. QJM 2003;96:635-642.

5 Gnanadesigan N, Espinoza RT, Smith R, Israel M, Reuben DB: Interaction of serotonergic antidepressants and opioid analgesics: is serotonin syndrome going undetected? J Am Med Dir Assoc 2005;6:265-269.

6 Gillman PK: Serotonin syndrome: history and risk. Fundam Clin Pharmacol 1998;12:482-491.

7 Isbister GK, Bowe SJ, Dawson A, Whyte IM: Relative toxicity of selective serotonin reuptake inhibitors (SSRIs) in overdose. J Toxicol Clin Toxicol 2004;42:277-285.

8 Bijl D: The serotonin syndrome. Neth J Med 2004;62:309-313.

-9 Sun-Edelstein C, Tepper SJ, Shapiro RE: Drug-induced serotonin syndrome: a review. Expert Opin Drug Saf 2008;7:587-596.

10 Mason PJ, Morris VA, Balcezak TJ: Serotonin syndrome. Presentation of 2 cases and review of the literature. Medicine 2000;79:201-209.

11 Egan TD, Sharma A, Ashburn MA, Kievit J, Pace NL, Streisand JB: Multiple dose pharmacokinetics of oral transmucosal fentanyl citrate in healthy volunteers. Anesthesiology 2000;92:665-673.

12 Ailawadhi S, Sung KW, Carlson LA, Baer MR: Serotonin syndrome caused by interaction between citalopram and fentanyl. J Clin Pharmacol 2007;32:199-202.

13 Rastogi R, Swarm RA, Patel TA: Case scenario: opioid association with serotonin syndrome. Anesthesiology 2011;115:1291-1298.

-14 Alkhatib AA, Peterson KA, Tuteja AK: Serotonin syndrome as a complication of fentanyl sedation during esophagogastroduodenoscopy. Dig Dis Sci 2010;55:215-216.

$\$ 15$ Riley TR 3rd: Predictors of pain with liver biopsy. Dig Dis Sci 2002;47:2151-2153. 